\title{
It's not a bug, it's boredom: Effortful willpower balances exploitation and exploration
}

\author{
Maik Bieleke ${ }^{1} \&$ Wanja Wolff',3
}

${ }^{1}$ Department of Developmental and Educational Psychology, Faculty of Psychology, University of Vienna, Universitätsstrasse 7, A-1010 Vienna, Austria

Phone: +43-1-4277-47404, Email: maik.bieleke@univie.ac.at

https://bildung-psy.univie.ac.at/en/about-us/maik-bieleke/

${ }^{2}$ Department of Sports Science, University of Konstanz, Universitätsstrasse 10, D-78457

Konstanz, Germany

Phone: +49-7531 88-3535, Email: wanja.wolff@uni-konstanz.de

https://scikon.uni-konstanz.de/persons/profile/wanja.wolff/

${ }^{3}$ Department of Educational Psychology, University of Bern, Fabrikstrasse 8, CH-3012 Bern, Switzerland

\begin{abstract}
The continuous revaluation of rewards lies at the core of Ainslie's account of willpower. Yet, he does not explicate the underlying experiential mechanisms. We draw upon theoretical, neuroscientific, and computational evidence to demonstrate that boredom evokes revaluation. By biasing behavior towards exploration, boredom necessitates effortful willpower to balance it against exploitation, thereby rendering suppression a highly adaptive function of willpower.
\end{abstract}

This manuscript has been submitted to Behavioral and Brain Sciences and is currently in press. Please cite it as follows:

Bieleke, M., \& Wolff, W. (2021). It's not a bug, it's boredom: Effortful willpower balances exploitation and exploration. Behavioral and Brain Sciences.

https://doi.org/10.1017/S0140525X20001053 
In the target article, Ainslie differentiates between effortless ("resolve") and effortful ("suppression") functions of willpower. Our focus is on suppression, which is thought to stabilize ongoing behavior against revaluations instigated by hyperbolic discounting. Crucially, suppression is conceived as a fragile and costly mechanism that needs to be "stiffened by resolve." Here, we argue that hyperbolic discounting and the fragility and costliness of suppression are both adaptive features that aid humans in navigating exploration-exploitation tradeoffs. Drawing upon theoretical, neuroscientific, and computational evidence, we argue that boredom is an experiential mechanism that drives revaluation and interacts with suppression in orienting goal-directed behavior.

Incidentally, boredom has already been discussed in the context of willpower (e.g., Ainslie, 2013), but only as one mechanism among many and without a dedicated theoretical framework that explicates its unique functional relevance: (1) boredom instigates the revaluation of potential rewards, (2) increases the costs of the resulting suppression, (3) and thereby biases behavior away from exploitation and towards exploration. Thus, we extend Ainslie's proposal by explicating boredom as a powerful mechanism that drives hyperbolic discounting and by highlighting why suppression is a highly adaptive mechanism that has consequently been favored by evolution.

Ainslie identifies hyperbolic discounting as an "inborn psychophysical tendency" that manifests itself in the dynamic revaluation of rewards. However, while he is explicit about the experiential mechanism that tracks the temporal dynamics of task-induced costs (i.e., effort), the target article remains silent on the mechanisms that underly revaluation. One ubiquitous experience linked to revaluations by recent work on willpower is boredom (Wolff \& Martarelli, 2020). Boredom emerges in situations that are perceived as meaningless and/or as misfitting one's mental resources (Westgate \&Wilson, 2018). Its experience serves as a dynamic (Mills \& Christoff, 2018), functional signal that an ongoing behavior decreases in value, prompting people to seek more rewarding alternative behaviors (Bench \& Lench, 2019). In line with this, neuroscientific research has shown that boredom, but not suppression, increases reward sensitivity (Milyavskaya et al., 2019). This logic can be extended to long-term goals, whose pursuit should then decrease in value relative to alternative goals that promise immediate gratification. Thus, boredom can be assumed to instigate the exact revaluations that underly hyperbolic discounting, which may lead to impulsive behaviors that must be suppressed to avoid what willpower research generally refers to as self-control failure. Consequently, boredom directly contributes to the demand for suppression (Wolff \& Martarelli, 2020).

In line with the literature, Ainslie argues that effort serves as a dynamic signal to quantify the ongoing costs of suppression (Shenhav et al., 2017). He attributes these costs to the need for "continuous vigilance against impulses" and suggests that "wastes of time do not typically feel effortful." While we agree with the first, we object to the latter assertion. Boredom does not constitute an affectively neutral signal; instead, it is an aversive sensation that increases the effort to continue with a course of action (Eastwood et al., 2012). Therefore, we argue that experiencing boredom does contribute to the costs of suppression by making it more effortful to persist (for initial experimental evidence, see Bieleke et al., 2020) - up to the point that people even become willing to trade boredom for pain (Wilson et al., 2014). Consequently, boredom not only devalues the pursuit of ongoing (long-term) goals, it simultaneously increases the costs of suppressing the pursuit 
of alternative (short-term) goals. This twofold effect makes the experience of boredom a powerful mechanism behind the disengagement from goal pursuit. Neuroscientific evidence provides tentative support for the implied interplay of boredom and suppression: Boredom has been linked to activation changes in the ventromedial prefrontal cortex (Mathiak et al., 2013), an area that is involved in valuation processes (Gläscher et al., 2009) and that plays a key role in indicating that a change in behavior is required (Domenech \& Koechlin, 2015). Crucially, information from such valuation areas is integrated by the dorsal Anterior Cingulate Cortex, whose role in specifying control commands and in relaying those commands to executive areas like the lateral Prefrontal Cortex is well established (Shenhav et al., 2013). Thus, the ventromedial Prefrontal Cortex's sensitivity towards rewards and the dorsal Anterior Cingulate Cortex's role in weighing prospective rewards against the costs of suppression is in line with the proposed twofold effect boredom has on goal pursuit.

Understanding boredom in terms of a functional signal that facilitates the disengagement from ongoing goal pursuit - by instigating revaluation and feeding into the costs of suppression - raises an important question: Are the fragility and the costliness of suppression undesirable properties? We argue they are not: these very properties allow for suppression to assume the role of flexibly balancing exploration against exploitation. Computational work has shown that boredom facilitates an intelligent system's ability to explore the environment (Gomez-Ramirez \& Costa, 2017). This shift from the longstanding and exclusive focus on prediction error minimization is in line with empirical (Geana et al., 2016) and theoretical work (Wolff \& Martarelli, 2020) on the role of boredom in driving exploration. Willpower by resolve, which is a function that favors long-term effortless goal pursuit (for an example, see Bieleke et al., in press), is not designed to adaptively account for boredom-induced impulses to explore. Instead, a more fragile mechanism like suppression is better suited to respond adequately to the dynamic changes in the costs and benefits of ongoing goal pursuit; it thereby provides degrees of freedom for flexibly balancing exploration against exploitation. This functional role of suppression as a fragile and costly mechanism might explain why evolution has favored imperfect self-control (Hayden, 2019).

\section{Conflicts of Interest}

The authors declare that there is no conflict of interest.

\section{Funding Statement}

This research received no specific grant from any funding agency, commercial or not-forprofit sectors. 


\section{References}

Ainslie, G. (2013). Monotonous tasks require self-control because they interfere with endogenous reward. Behavioral and Brain Sciences, 36(6), 679-680. https://doi.org/10.1017/S0140525X13000915

Bench, S. W., \& Lench, H. C. (2019). Boredom as a seeking state: Boredom prompts the pursuit of novel (even negative) experiences. Emotion, 19(2), 242-254. https://doi.org/10.1037/emo0000433

Bieleke, M., Barton, L., \& Wolff, W. (2020). Trajectories of boredom in self-control demanding tasks. PsyArXiv. https://doi.org/10.31234/osf.io/ekqrv

Bieleke, M., Keller, L., \& Gollwitzer, P. M. (in press). If-then planning. European Review of Social Psychology.

Domenech, P., \& Koechlin, E. (2015). Executive control and decision-making in the prefrontal cortex. Current Opinion in Behavioral Sciences, 1, 101-106.

https://doi.org/10.1016/j.cobeha.2014.10.007

Eastwood, J. D., Frischen, A., Fenske, M. J., \& Smilek, D. (2012). The unengaged mind: Defining boredom in terms of attention. Perspectives on Psychological Science, 7(5), 482-495. https://doi.org/10.1177/1745691612456044

Geana, A., Wilson, R., Daw, N., \& Cohen, J. (2016). Boredom, information-seeking and exploration. In A. Papafragou, D. Grodner, D. Mirman, \& J. C. Trueswell (Eds.), Proceedings of the $38^{\text {th }}$ annual conference of the Cognitive Science Society (pp. 17511756). Cognitive Science Society.

Gläscher, J., Hampton, A. N., \& O’Doherty, J. P. (2009). Determining a role for ventromedial prefrontal cortex in encoding action-based value signals during reward-related decision making. Cerebral Cortex, 19(2), 483-495. https://doi.org/10.1093/cercor/bhn098

Gomez-Ramirez, J., \& Costa, T. (2017). Boredom begets creativity: A solution to the exploitation-exploration trade-off in predictive coding. Biosystems, 162, 168-176. https://doi.org/10.1016/j.biosystems.2017.04.006

Hayden, B. Y. (2019). Why has evolution not selected for perfect self-control? Philosophical Transactions of the Royal Society B: Biological Sciences, 374(1766), 20180139. https://doi.org/10.1098/rstb.2018.0139

Mathiak, K. A., Klasen, M., Zvyagintsev, M., Weber, R., \& Mathiak, K. (2013). Neural networks underlying affective states in a multimodal virtual environment: Contributions to boredom. Frontiers in Human Neuroscience, 7, 820.

https://doi.org/10.3389/fnhum.2013.00820

Mills, C., \& Christoff, K. (2018). Finding consistency in boredom by appreciating its instability. Trends in Cognitive Sciences, 22(9), 744-747.

https://doi.org/10.1016/j.tics.2018.07.001

Milyavskaya, M., Inzlicht, M., Johnson, T., \& Larson, M. J. (2019). Reward sensitivity following boredom and cognitive effort: A high-powered neurophysiological investigation. Neuropsychologia, 123, 159-168. https://doi.org/10.1016/j.neuropsychologia.2018.03.033 
Shenhav, A., Botvinick, M. M., \& Cohen, J. D. (2013). The expected value of control: An integrative theory of anterior cingulate cortex function. Neuron, 79(2), 217-240. https://doi.org/10.1016/j.neuron.2013.07.007

Shenhav, A., Musslick, S., Lieder, F., Kool, W., Griffiths, T. L., Cohen, J. D., \& Botvinick, M. M. (2017). Toward a rational and mechanistic account of mental effort. Annual Review of Neuroscience, 40, 99-124. https://doi.org/10.1146/annurev-neuro-072116-031526

Westgate, E. C., \& Wilson, T. D. (2018). Boring thoughts and bored minds: The MAC model of boredom and cognitive engagement. Psychological Review, 125(5), 689-713.

https://doi.org/10.1037/rev0000097

Wilson, T. D., Reinhard, D. A., Westgate, E. C., Gilbert, D. T., Ellerbeck, N., Hahn, C., Brown, C. L., \& Shaked, A. (2014). Just think: The challenges of the disengaged mind. Science, 345(6192), 75-77. https://doi.org/10.1126/science.1250830

Wolff, W., \& Martarelli, C. S. (2020). Bored into depletion? Toward a tentative integration of perceived self-control exertion and boredom as guiding signals for goal-directed behavior. Perspectives on Psychological Science, 15(5), 1272-1283.

https://doi.org/10.1177/1745691620921394 